\title{
A study of Edge Detection Techniques for Segmentation Computing Approaches
}

\author{
S.Lakshmi \\ Jeppiar Engineering College \\ Chennai, India
}

\author{
Dr.V.Sankaranarayanan \\ Crescent Engineering College \\ Chennai, India
}

\begin{abstract}
Edge is a basic feature of image. The image edges include rich information that is very significant for obtaining the image characteristic by object recognition. Edge detection refers to the process of identifying and locating sharp discontinuities in an image. So, edge detection is a vital step in image analysis and it is the key of solving many complex problems. In this paper, the main aim is to study the theory of edge detection for image segmentation using various computing approaches based on different techniques which have got great fruits.
\end{abstract}

\section{General Terms}

Pattern Recognition, Digital Image Processing, Algorithms.

\section{Keywords}

Image Segmentation, Edge detection, Fuzzy logic, Genetic Algorithm, Neural network, Mathematical morphology, Wavelet Transform.

\section{INTRODUCTION}

The separation of the image into object and background is a critical step in image interpretation. When we imitate the human visual system by using computer algorithms, quite a lot of problems can be encountered. Segmentation subdivides an image into its constituent regions or objects. The level to which the subdivision is carried depends on the problem being solved. That is, segmentation should stop when the objects of interest in an application have been isolated [1][2]. Color image segmentation is usually the first task of any image analysis process. All subsequent tasks such as edge detection, feature extraction and object recognition rely heavily on the quality of the segmentation. Without a good segmentation algorithm, an object may never be recognizable. However, in many cases parts of contours can be correctly reconstructed either by performing edge grouping or as parts of boundaries of segmented regions. Therefore, recognition of objects based on their contour parts seems to be a promising as well as a necessary research direction. The latest survey on color image segmentation techniques discussed the advantages and disadvantages of classical segmentation techniques, such as histogram thresholding, clustering, edge detection and region based methods, vector based, fuzzy techniques as well as physics based methods [3]. Over segmenting an image will split an object into different regions while under segmenting it will group various objects into one region. In this way the segmentation step determines the eventual success or failure of the analysis. For this reason, considerable care is taken to improve the state of the art in color image segmentation. Extensive research has been done in creating many different approaches and algorithms for image segmentation, but it is still difficult to assess whether one algorithm produces more accurate segmentations than another, whether it be for a particular image or set of images, or more generally, for a whole class of images. Every year new edge detection algorithms are published. This paper analysis some recent approaches for detecting edges for segmentation.

This paper is organized as follows. Section 2 is for the purpose of providing some information about edge detection for image segmentation. Section 3 is focused on showing the challenges in edge detection and edge classification methods. Section 4 explains different computing approaches to edge detection. Section 5 concentrates on comparison of various edge detection methods. Section 6 presents the conclusion.

\section{EDGE DETECTION FOR IMAGE SEGMENTATION}

The effectiveness of many image processing and computer vision tasks depends on the perfection of detecting meaningful edges. Edge detection has been a challenging problem in low level image processing. It becomes more challenging when color images are considered because of its multi dimensional nature. Color images provide accurate information about the object which will be very useful for further operations than gray scale images. Due to some unavoidable reasons such as distortion, intensity variation, noise, segmentation errors, overlap (large number of distracting objects i.e., clutter), and occlusion of objects in digital images, it is usually impossible to extract complete object contours or to segment the whole objects. Due to lack of object edge information the output image is not visually pleasing. A huge number of methods are available in the literature to segment images. This task is hard and very important, since the output of an image segmentation algorithm can be fed as input to higher-level processing tasks, such as model-based object recognition systems.

There are many techniques in the literature used for edge detection some of them are based on error minimization, maximizing an object function, fuzzy logic, wavelet approach, morphology, genetic algorithms, neural network and Bayesian approach.

Color image segmentation techniques can be roughly classified into four types such as histogram based approaches, neighborhood based approaches, clustering based approaches and hybrid based approaches. Histogram thresholding is widely accepted and easily computable technique in which the images are composed of regions with different gray level ranges. The 
main disadvantage of this technique is the lack of spatial relationship information of the pixels. The neighborhood based approach applies the uniformity criteria to segment the image i.e., the neighboring pixels within the region should have similar values in intensity, color or texture. E.g. Region based techniques. Clustering based approach uses a fuzzy logic to define membership of the pixels. Regions are formed by inspecting the membership values of pixels using partition method e.g. Fuzzy C-means (FCM) algorithm. Hybrid based techniques improve the segmentation result by combining all above methods for segmentation.[4].

\subsection{Types of Edges}

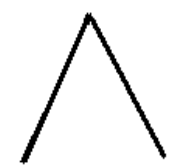

Convex root edge

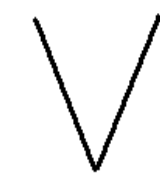

Concave roof edge

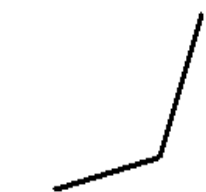

Concave ramp edge

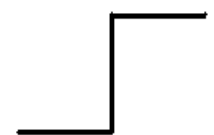

Step edge

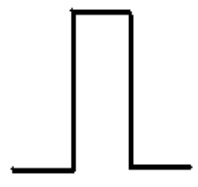

Bar edge
Figure.1 Various types of edges

\subsection{General steps in Edge Detection}

Generally, Edge detection contains three steps namely Filtering, Enhancement and Detection.

i. Filtering: Some major classical edge detectors work fine with high quality pictures, but often are not good enough for noisy pictures because they cannot distinguish edges of different significance. Noise is unpredictable contamination on the original image. There are various kinds of noise, but the most widely studied two kinds are white noise and "salt and pepper" noise. In salt and pepper noise, pixels in the image are very different in color or intensity from their surrounding pixels; the defining characteristic is that the value of a noisy pixel bears no relation to the color of surrounding pixels. Generally this type of noise will only affect a small number of image pixels. When viewed, the image contains dark and white dots, hence the term salt and pepper noise. In Gaussian noise, each pixel in the image will be changed from its original value by a small amount. Random noise to describe an unknown contamination added to an image. To reduce the influence of noise, Marr suggested filtering the images with the Gaussian before edge detection. ii. Enhancement: Digital image enhancement techniques are concerned with improving the quality of the digital image. The principal objective of enhancement techniques is to produce an image which is better and more suitable than the original image for a specific application. Linear filters have been used to solve many image enhancement problems. Throughout the history of image processing, linear operators have been the dominating filter class. Not all image sharpening problems can be satisfactorily addressed through the use of linear filters. There is a need for nonlinear geometric approaches, and selectively in image sharpening is the key to its success. A powerful nonlinear methodology that can successfully address the image sharpening problem is mathematical morphology. [5]

iii. Detection: Some methods should be used to determine which points are edge points or not.

\section{CHALLENGES IN CLASSIFICATION AND DETECTION METHODS}

Extraction and segmentation has to deal with the following challenges:

(1)The changes in lighting conditions

(2) The background is dynamic

(3) Luminance and geometrical features,

(4) Noise volume has a great impact on shaping the edge.

(5) Missing to detect existing edges

(6) Detecting edges where it does not exist (false edge) and

(7) Position of the detected edge to be shifted from its true location (shifted edge or dislocated edge).

\subsection{Classification of edges and its Detection methods}

The classification of the edge detection algorithms based on the behavioral study of edges with respect to the operators.

- Classical or Gradient based edge detectors (first derivative )

- Zero crossing (second derivative)

- Laplacian of Gaussian (LoG)

- Gaussian edge detectors

- Colored edge detectors

\subsection{Classical Edge detectors}

It contains classical operators and uses first directional derivative operation. Sobel (1970), Prewitt (1970), Krisch (1971), Robinson(1977), Frei-Chen(1977). Detection of edges and their orientation is the main advantage of these types of edge detectors. Main disadvantage of these types of edge detectors are sensitive to noise and inaccurate.

\subsubsection{The Roberts Detection}

In Robert cross algorithm the horizontal and vertical edges bring out individually and then they put together for the resulting edge detection. 


\begin{tabular}{|c|c|}
\hline+1 & 0 \\
\hline 0 & -1 \\
\hline
\end{tabular}

$\mathrm{G}_{\mathrm{x}}$

\begin{tabular}{|c|c|}
\hline 0 & +1 \\
\hline-1 & 0 \\
\hline
\end{tabular}

$\mathrm{G}_{\mathrm{y}}$
The two individual images $\mathrm{Gx}$ and Gy are combined using the approximation equation $|\mathrm{G}|=|\mathrm{Gx}|+|\mathrm{Gy}|$ or by using $\mathrm{G}=$ sqrt $(\mathrm{Gx} * \mathrm{Gx}+\mathrm{Gy} * \mathrm{~Gy})$ to get the exact magnitude values. As the Roberts Cross kernels are relatively small, they are highly susceptible to noise.

\subsubsection{Sobel Edge Detection}

The Sobel edge detection technique is similar to that of the Roberts Cross algorithm. Despite the design of Sobel and Robert are common, the main difference is the kernels that each uses to obtain the image is different. The sobel kernels are more suitable to detect edges along the horizontal and vertical axis whereas the Roberts's able to detect edges run along the vertical axis of $45^{\circ}$ and $135^{\circ}$.

\begin{tabular}{|c|c|c|}
\hline+1 & +2 & +1 \\
\hline 0 & 0 & 0 \\
\hline-1 & -2 & -1 \\
\hline
\end{tabular}

\begin{tabular}{|c|c|c|}
\hline-1 & 0 & +1 \\
\hline-2 & 0 & +2 \\
\hline-1 & 0 & +1 \\
\hline
\end{tabular}

\subsubsection{Zero Crossing}

It uses second derivative and it includes Laplacian operator. It is having fixed characteristics in all directions and sensitive to noise. Haralick proposed the use of zero-crossing of the second directional derivative of the image intensity function.

\subsubsection{Laplacian of Gaussian (LoG)}

It was invented by Marr and Hildreth (1980). The Gaussian filtering is combined with Laplacian to break down the image where the intensity varies to detect the edges effectively.

\subsubsection{Gaussian Edge Detectors}

Since it reduces the noise by smoothing the image, it gives better results in noisy environment. The noteworthy operators are Canny and ISEF (Shen-Castan). It is very time consuming and very complex for computation.

\subsubsection{Canny Edge Detector}

The popular edge detection algorithm Canny first presented in 1986.The problem with this type of traditional edge detection approach is that a low threshold produces false edges, but a high threshold misses important edges. First requires that the image be smoothed with a Gaussian mask, which cuts down significantly on the noise within the image. Then the image is run through the Sobel algorithm, and as discussed before, this process is hardly affected by noise. Lastly, the pixel values are chosen based on the angle of the magnitude of that pixel and its neighboring pixels. Unlike Roberts Cross and much like Sobel, the canny operation is not very susceptible to noise. If the Canny detector worked properly it would be superior to both Sobel and
Roberts Cross, the only drawback is that it takes longer to compute.

\section{DIFFERENT APPROACHES}

\subsection{Fuzzy based approach}

In 1990's, Sinha and Dougherty import fuzzy mathematics into morphology and forming fuzzy mathematical morphology. Image information is very complex, and image process may not be integrated or accurate, so fuzzy set theory used in image analysis can get better effect than other computing methods.

Dong Hu and Zianzhong Tian in their paper, they integrate the multi-directions characteristic of structure elements and image fuzzy characteristic into mathematical morphology and then detect the edges using mathematical morphology [6. ]

Using fuzzy reasoning we can splendidly to enhance and detect edges. Fuzzy set theory is an approximation tool in modeling ambiguity or uncertainty and has been applied in image processing. Using some fuzzification function we can very well remove the noise efficiently from the given image.

George and Madan proposed fuzzy uncertainty measures for image processing in 1994.They showed that the fuzzy uncertainty measure is an approach for representing, processing and quantitatively evaluating the information in gray- tone images. A gray-tone image can be transformed into a fuzzy image by a fuzzification function. Using Mathematical morphology operator's dilation and erosion, the fuzzification functions for positive edge uncertainty and negative edge uncertainty is defined. [7]

In the Fuzzy reasoning system, the rules are framed for edge localization and the rules are based on the following simple reasons. If a pixel $(i, j)$ possesses larger edge uncertainty, this point should be preserved. Inversely, if it possesses smaller edge uncertainty, this point is regarded as a non-edge point.

Kanchan Deshmukh and Ganesh presented a neuro-fuzzy system to perform multilevel segmentation of color images in HSV color space. It has two stages. In fist stage, the number of clusters of pixels with similar color is found using FMMN clustering algorithm. In the second stage, neural network is used to find multiple objects in the image. [8]

ACISFMC system consists of a multilayer neural network which performs adaptive, multilevel thresholding of the color image. Fuzzy entropy is used as a tool to measure the error of the system. Adaptive threshold selection block is responsible to determine clusters and compute a multilevel sigmoid function of neurons. Neural network segmentation block does the actual segmentation based on the number of objects found out by adaptive threshold selection block.

The gradient and standard deviation computed at each pixel and are used as fuzzy system input. The fuzzy system includes member functions and fuzzy rules which decide about pixel classification as edge or non-edge. [9]. 


\subsection{Wavelet approach}

Very first wavelet analysis comes for existence in late 1980's.Since wavelet based on mathematics the wavelet transform is the representation of functions of wavelets. Even though the Fourier analysis is a good enough tools for frequency analysis, but it is not dependent of time and it is not able to describe the local properties of functions in terms of their spectral properties.

Wavelet transforms are classified into discrete wavelet transforms (DWTs) and continuous wavelet transforms (CWTs). The Discrete Wavelet Transform (DWT) has been a successful technique used in edge detection.

First, an image is decomposed using an ordinary, isotropic and separable wavelet transform. For this category of wavelet, the geometry of the wavelet is the same regardless of direction (rotational invariance) and is strongly vertical and horizontal in nature. Two examples of an isotropic wavelet are Gaussian or Mexican Hat. The 2-D discrete wavelet transform decomposes the image into sub-images, 3 details and 1 approximation. The approximation looks just like the original; only on $1 / 4$ the scale. The 2-D DWT is an application of the 1-D DWT in both the horizontal and the vertical directions. The DWT separates an image into a lower resolution approximation image (LL) as well as horizontal (HL), vertical (LH) and diagonal (HH) detail components. The low-pass and high-pass filters of the wavelet transform naturally breaks a signal into similar (low-pass) and discontinuous rapidly changing (high-pass) sub-signals, utilizing both basic properties. It is this characteristic that supremely suits the DWT for edge detection. [10].

A directional wavelet such as Gabor (Morlet) and Cauchy combined with Otsu's single thresholding method is used to detect whether the pixel belongs to an edge or not. [24]. Haar Wavelet is a type of wavelet which is also used to find edges in an efficient manner. The work of Jung and Scharcanski, which is largely based on combining the watershed method with the DWT. Watershed segmentation, splits an image into regions using a geographical analogy for gray-scale images. The objective of the watershed transform process is to convert an original image into a second image in which the features that are of significance become the basins.

The isotropic wavelets do extremely well at isolating point wise discontinuities and the directional wavelets excel at locating contoured paths. The directional wavelet can correct the weakness of isotropic wavelets and vice versa. Hence, isotropic and directional wavelets can combine together to detect edges in well organized manner.

Generally the edge detection approaches have multi-step process such as preprocessing, noise cleaning, thresholding coefficients, smoothing, post processing. Every step may be performed by using DWT with multiple iterations. Therefore the DWT is combined with various other methods for an optimal solution for the edge detection problem.

\subsection{Genetic Algorithm Approach}

One of the most recent interesting $\mathrm{AI}$ areas in the image feature extraction regards the Evolutionary Algorithms (EAs). Commonly, these kinds of algorithms use a heuristic approach to find approximate solutions to optimization and search problems. The EAs are inspired by Darwin's theory about biological evolution. Indeed, there are several kinds of EAs (Freitas, 2002; Korolev, 2007; Zheng et al., 2007), such as: Genetic Algorithms (GAs), Genetic Programming (GP), Evolutionary Programming (EP), Evolution Strategies (ESs), and others.

Genetic algorithms (GA) are random search algorithms based on the theory of biological evolution. These algorithms require an initial population of individuals, which are representatives of possible solutions of the problem being solved. The population evolves by transformations applied to its individuals while a fitness function is used to determine the strength of the elements in the population. The elements of the population are known as chromosomes and are represented by strings of bits. The fitness function is usually computed with basis on the values of those bits. An iteration of the algorithm is basically equivalent to a generation in the evolutionary process. [11]

Mainly, a genetic algorithm consists of three most important operations. They are Selection, Crossover and Mutation.

Selection: Fitness-proportional selection-The chromosome with minimum fitness value and another randomly chosen chromosome are selected from the parent pool to process crossover and mutation.

Crossover: The crossover recombines two individuals to have new ones which might be better.

Mutation: The mutation procedure introduces random changes in the population in order to steer the algorithm from local minimums that could prevent the discovery of the global solutions to the problem. [12]

The GA is started with a set of abstract candidate solutions population. These solutions are represented by chromosomes (genotypes or genomes). The Solutions from one population are taken and used to form a new population. This last process is motivated by the hope that the new population will be better than the old one. In each generation, the fitness of each candidate solution is evaluated, multiple candidate solutions are stochastically selected from the current solutions (based on their fitness), and modified (recombined and/or mutated and/or others "genetic" operations) to form a new population of candidate solutions. The new population is then used in the next iteration of the algorithm. GENIE (GENetic Imagery Exploration) (Perkins et al., 2000) uses a genetic programming approach to produce automatic feature extraction (AFE) tools for broad-area features in multispectral, hyper spectral, panchromatic, and multi-instrument imagery. Another interesting application, mentioned in (Del Buono, 2007) is Face Recognition ActiveX DLL. 


\subsection{Neural network}

Neural networks are nothing but the computer algorithms contend with how the way the information is processed in nervous system. Neural network diversifies from other artificial intelligence technique by means of the learning capacity.

Digital images are segmented by using neural networks in two step process. First step is pixel classification that depends on the value of the pixel which is part of a segment or not. Second step is edge detection that is the detection of all pixels on the borders between different homogeneous areas which is part of the edge or not [13]. Several neural networks are available in the literature for edge detection. The potential base function for digital image processing can be created using differential operators.

Generally, the neural network consists of three layers such as input layer, hidden layer and output layer as in the fig [2]. Each layer consists of fixed number of neurons equal to the number of pixels in the image. The activation function of neuron is a multi sigmoid. The major advantage of this technique is that, it does not require a priori information of the image. The number of objects in the image is found out automatically.

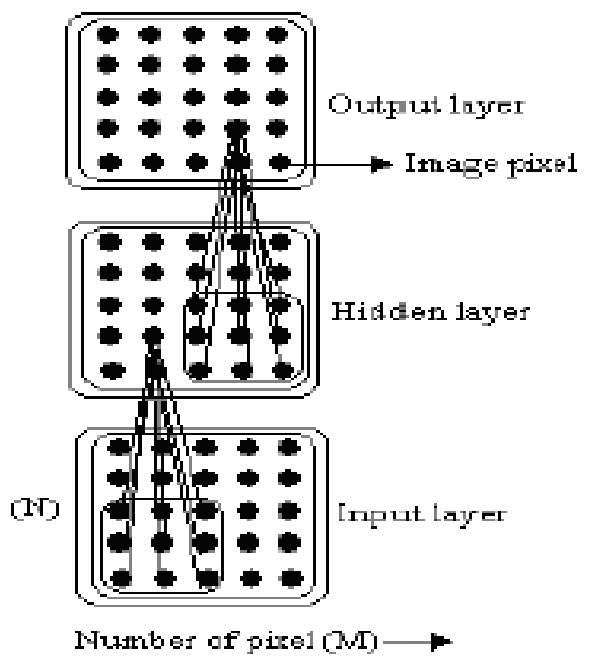

Figure.2 .Architecture of Neural Network

The feed-forward error-back propagation neural networks are trained with $3 \times 3$ to $11 \times 11$ inputs, 4 to 8 units in the single hidden layer, and a single output. All units used sigmoid activation functions. [13]. Hidden nodes, whose weights were regarded as a template, which is similar to any image filter (e.g. Kirsch or Sobel templates), and its Taylor series coefficients, were used to analyze the order of this template. Interesting results were found when some small neural networks edge detectors were trained with sharp edges whilst others were trained with sharp, blurred, and noisy variants of the same images. [14]

Each neuron is associated with a number for the neuron's activation. Each connection in the network is associated with a weight. The activation functions used for neuron outputs can be either linear or non-linear. The input signal is processed through input layer, hidden layer and output layer. The status of neurons in every layer affects status of neurons in the next layer only.[15]. Neurons in the same layer do not have connection among themselves. The output of the nodes in one layer is transmitted to the nodes in another layer via links that amplify or inhibit such outputs through weighting factors. Except for the input layer nodes, the total input to each node is the sum of weighted outputs of the nodes in the previous layer. Each node is activated in accordance with the input to the node and the activation function of the node.

Wavelet neural network (WNN) based on the wavelet transform theory, is a novel, multiresolution, hierarchical artificial neural network, which originally combines the good localization characteristics of the wavelet transform theory and the adaptive learning virtue of neural networks.[16].The first module extracts the feature and the second one is WNN classifier which is used to locate the position of the edges.

A three-layer neutral network is employed to determine the structure elements in the morphology method, so that the image can be smoothened and all probable edge points can be detected. Zernike moments operator is adopted to locate the edge to subpixel accuracy degree.[17]

The system consists of a neural network that performs the segmentation using multilevel thresholding activation function. The main advantage of this method is that, it segments the color image without priori knowledge of the image. The threshold and target values are used to construct an activation function of neuron. The error of the system is calculated and back propagated to change the weights of neural network. This process continues until a minimum error is achieved. The output of the system at this stage is a colored segmented image. [18]

The ANNSCIS (Adaptive Neural Network System for Color Image Segmentation) uses the HSV model for color image segmentation. The adaptive thresholding block is responsible to find out the number of clusters and computation of multi-level sigmoid function for the neurons in the neural network. At every training epoch, the error is calculated by taking the difference between actual output and desired output of neuron. Here desired output of the neuron is its target value. Once the error calculated, it is back propagated to update the weights. The aim of neural network system is to minimize the error to obtain a segmented image. This training process continues until a minimum error is achieved. The output of the network is colored segmented image.

\subsection{Morphology}

Being based on Set theory of mathematics, it slowly grows up a new theory and innovative method for digital image processing and recognition. The morphological edge detection algorithm is developed from the basic morphological operations. 
Morphological operators are directly deal with the shape information with the help of a structuring element.

We can organize images into two sets.(i) Binary images (ii)grey level and color images. The binary images are two valued one that may be 0 or 1 .The grey level images, the pixel value may be any integer between 0 and some value. Color image is an extension of grey level image where the values at each pixel represented by a vector of three elements with respect to red, green and blue components of color information.

The most basic operations are dilation and erosion which may be defined by using union and intersection. Dilation increases the object and erosion shrinks the object. Using the basic operators' dilation and erosion, two more operators are defined. They are Opening and Closing. Opening retains only those parts of the objects that can fit in the structuring element. Closing fills up small holes and gulfs. Thus they both can extract fine shape features that are narrower than the structuring element.

A binary image is " 0 " set which representing background and " 1 " set representing object. Structuring element is a smaller set compared to the image. The dilation of image A by structuring element $\mathrm{B}$, denoted by $\mathrm{A} \oplus \mathrm{B}$, is define as

$$
\mathrm{A} \oplus \mathrm{B}=\{\mathrm{x} \mid \mathrm{x}=\mathrm{a}+\mathrm{b} \text {, for some } \mathrm{a} \in \mathrm{A} \text { and } \mathrm{b} \in \mathrm{B}\}
$$

The erosion of $\mathrm{A}$ by $\mathrm{B}$, denoted by $\mathrm{A} \Theta \mathrm{B}$, is defined as

$$
\mathrm{A} \Theta \mathrm{B}=\{\mathrm{x} \mid \mathrm{b}+\mathrm{x} \in \mathrm{A}, \forall \mathrm{b} \in \mathrm{B}\}
$$

The morphological opening of $\mathrm{A}$ and $\mathrm{B}$, denoted by $\mathrm{A} \circ \mathrm{B}=(\mathrm{A} \Theta \mathrm{B}) \oplus \mathrm{B}$.

The morphological closing of $\mathrm{A}$ by $\mathrm{B}$, denoted by $\mathrm{A} \bullet \mathrm{B}=(\mathrm{A} \oplus \mathrm{B}) \Theta \mathrm{B}$.

The hit-or-miss transformation of A by B is denoted by

$$
\mathrm{A} \otimes \mathrm{B}=(\mathrm{A} \Theta \mathrm{B}) \cap\left(\mathrm{A}^{c} \Theta \mathrm{B}_{2}\right) \text { where } \mathrm{B} \text { is } \mathrm{a}
$$

structuring element and $\mathrm{A}^{c}$ is the complement of $\mathrm{A}$. Mathematical morphology operations can be combined with many other methods such as neural network, Fuzzy reasoning, wavelet, watershed algorithm, genetic algorithm etc to the search for specific signal and image processing tasks.

\section{COMPARISON OF EDGE DETECTION METHODS}

It is a very challenging problem to evaluate edge detection results produced by various edge detectors with different parameters. The performance of the edge detector is compared to commonly used or comparable algorithms such as the Canny Sobel and Robert's edge detection algorithms.

Extensive research has been done in creating many different approaches and algorithms for image segmentation, but it is still difficult to assess whether one algorithm produces more accurate segmentations than another, whether it be for a particular image or set of images, or more generally, for a whole class of images.

Classical techniques for accurate detection of edge features, as exemplified by Canny operator, demands such expensive operations as the iterative use of Gaussians, Laplacians and their designs are largely sequential. Wavelet based edge detectors provide a facility for varying the scaling factor, which helps in differentiating the weak edges from strong edges.

Subjective methods borrowed from the field of psychology and use human judgment to evaluate the performance of edge detectors. On the other hand, objective methods use to measure the performance of edge detectors using signal to noise ratio and mean square error between the edge detectors images and the original one. Evaluation is done using both a Receiver Operating Characteristics (ROC) analysis and a Chi-square test, and considers the tradeoff between information and noisiness in the detection results. The best edge detector parameter set (PS) is then selected by the same statistical approach. Results are demonstrated for several edge detection techniques, and compared to published subjective evaluation results. Simulation results indicate that the proposed edge detector outperforms competing edge detectors and offers superior performance in edge detection in digital images corrupted by noise.

The most common method for evaluating the effectiveness of a segmentation method is subjective evaluation, in which a human visually compares the image segmentation results for separate segmentation algorithms, which is a tedious process and inherently limits the depth of evaluation to a relatively small number of segmentation comparisons over a predetermined set of images. Another common evaluation alternative is supervised evaluation, in which a segmented image is compared against a manually-segmented or pre-processed reference image. Evaluation methods that require user assistance, such as subjective evaluation and supervised evaluation, are infeasible in many vision applications, so unsupervised methods are necessary. Unsupervised evaluation enables the objective comparison of both different segmentation methods and different parameterizations of a single method, without requiring human visual comparisons or comparison with a manually-segmented or pre-processed reference image.

Additionally, unsupervised methods generate results for individual images and images whose characteristics may not be known until evaluation time. Unsupervised methods are crucial to real-time segmentation evaluation, and can furthermore enable self-tuning of algorithm parameters based on evaluation results.

Image quality measures (IQMs) are figures of merit used for the evaluation of imaging systems or of coding/processing techniques. Various image quality metrics are discussed in [25].There are two types of edge based quality measures, that is displacement of edges positions or their consistency across resolution levels explained in detail. They are Pratt edge measure and Edge stability measure. 


\section{CONCLUSION}

The purpose of this paper is to present a survey of various approaches for image segmentation based on edge detection techniques. In future, we plan to design a novel approach for edge detection and object recognition.

\section{REFERENCES}

[1] Rafael C. Gonzalex, Richard E. Woods, Image Segmentation in the book titled Digital Image Processing Chapter 10,Page No>589-664,Second Edition.

[2] Paulus, Color image processing: methods and applications in color image Segmentation Selected Techniques, chapter 5, pp.103-128, CRC Press, Boca Rato, Fla, USA, 2007.

[3] Most Recent Trends and Future Research Directions in Color in Image and video Processing, Hidwani publications, 2008.

[4] First Arkansan Deshmukh,Member IAENG,Second B.Ganesh Shinde, Adaptive Color Image Segmentation Using Fuzzy Min-Max Clustering, Engineering Letters, Advance online Publication,Aug-2006.

[5] T.M.Mahmoud and S.Marshall, Edge -Detected Guided Morphological Filter for Image Sharpening in the EURASIP Journal on Image and Video Processing, 2008

[6] Dong $\mathrm{Hu}$ and Zianzhong Tian, A Multi-directions Algorithm for Edge Detection Based on Fuzzy Mathematical Morphology, Proceeding of the $16^{\text {th }}$ International Conference on Artificial Reality and TelexistenceWorkshops (ICAT'06'), IEEE, 2006.

[7] Rey-Sern L ,Edge Detection by Morphological Operations and Fuzzy Reasoning, in, IEEE, 2008.

[8] First A.Kanchan Deshmukh,Member IAENG,Second B.Ganesh Shinde, Adaptive Color Image Segmentation Using Fuzzy Min-Max Clustering, Engineering Letters, Advance online Publication,Aug-2006.

[9] Wafe barkhoda,Fardin Akhlaqian Tab,Om-Kolsoom Shahryari, Fuzzy Edge Detection Based on Pixel's Gradient and Standard Deviation Values,Iran,2009.

[10] Evelyn Brannock, Michael Weeks, A synopsis of Recent Work in Edge detection using the DWT, in IEEE, 2008.

[11] Timothy P.Donovan and Nelson L.Passos, Edge Detection through the Use of a Combined Genetic Algorithm -Linear Technique Approach.
[12] M.K.Lee, S.W.Leung, T.L.Pun and H.L.Cheung, Edge Detection By Genetic Algorithm in IEEE, 2000.

[13] Berend jan van der Zwaag, Kees Slump and Lambert Spannenburg Analysis of Neural networks for Edge Detection in Proceedings IEEE Benelux Signal Processing Symposium,2002, Swedan.

[14] Maher I.RAJAB AND KHALID A.AL-HINDI, Analysis of Neural Network Edge Pattern Detectors in Terms of Domain Functions, SEAS Transactions on Information Science \& Applications “,issue 2, Volume 5, February 2008.

[15] Lihong Zheng and Xingjian He, Edge Detection Based on Modified BP Algorithm of ANN,2007, Australia.

[16] Nian Cai, A Wavelet Neural Network for Edge Detection, Congress on Image and Signal Processing, IEEE, 2008.

[17] Yu Lei and Nie Jiafa, Subpixel Edge Detection Based on Morphological Theory in the Proceedings of the World Congress on Engineering and Computer Science ,WCECS 2008, October ,2008,San Francisco, USA

[18] Kanchan Deshmukh, Abhijeet Nandedkar, Yeshwant Joshi and Ganesh Shinde, Multilevel Approach for Color Image Segmentation

[19] A.Jiang,C.L.Chuang,Y.L.Lu and C.S.Fahn, Mathematicalmorphology-based edge detectors for detection of thin edges in low-contrast regions in The Institution of Engineering and Technology (IET) Image Processing,pp.269-277,2007.

[20] Bhabatosh Chanda, Morphological Algorithms for Image Processing in IETE Technical Review, Vol.25, No-1, JanFeb, 2008.

[21] Zijuan Yu, Yuqian Zhao, XiaoFang Wang, Research Advances and Prospects of Mathematical Morphology in Image Processing in IEEE, 2008.

[22] Osslan Osiris Vergara Villegas and Raul Pinto Elias, Digital Image Processing in Wavelet Domain

[23] JunLi ,Thesis, A Wavelet Approach to Edge Detection, Sam Houston State University,Texas,2003.

[24] J. M. Niya, A. Aghagolzadeh , M. A. Tinati, and S.Feizi, 2 step wavelet-based edge detection using gabor and cauchy directional wavelets, IEEE Trans. Acoustics,Speech, Signal Processing, vol. 37, page(s): 2091-2110, December 1989.

[25] Ismail Avcibas, Bulent Sankar, Khalid Sayood, Statistical evaluation of image quality measures, Journal of Electronic Imaging ,2002. 